\title{
Philosophiques
}

\section{Spinoza et le problème de l'intentionnalité}

\section{Elhanan Yakira}

Volume 29, numéro 1, printemps 2002

Spinoza sous le prisme de son anthropologie

URI : https://id.erudit.org/iderudit/009569ar

DOI : https://doi.org/10.7202/009569ar

Aller au sommaire du numéro

Éditeur(s)

Société de philosophie du Québec

ISSN

0316-2923 (imprimé)

1492-1391 (numérique)

Découvrir la revue

Citer cet article

Yakira, E. (2002). Spinoza et le problème de l'intentionnalité. Philosophiques, 29(1), 139-146. https://doi.org/10.7202/009569ar d'utilisation que vous pouvez consulter en ligne.

https://apropos.erudit.org/fr/usagers/politique-dutilisation/ 


\title{
Spinoza et le problème de l'intentionnalité
}

\author{
ELHANAN YAKIRA \\ Université Hébraïque de Jérusalem \\ yakira@h2.hum.huji.ac.il
}

\begin{abstract}
RÉSUMÉ. - L'auteur propose une lecture de Spinoza à la lumière de la notion husserlienne d'intentionnalité. Il essaie de trouver un point commun aux deux systèmes afin de montrer la très grande originalité de la théorie de la vérité et de la signification de Spinoza qui rejette la primauté de la notion d'intentionnalité.

ABSTRACT. - This article proposes to read Spinoza in light of Husserl's notion of intentionality. It tries to find a common ground between the two systems, in order to show that Spinoza's theory of truth and of sense is highly original in its implicit rejection of the primordiality of the notion of intentionality.
\end{abstract}

Le sujet de cet article, Spinoza et l'intentionnalité, étonnera peut-être. À plus d'un titre, celui-ci pourra sembler incongru. Premièrement, la notion d'intentionnalité ne possède pas une signification unique déterminée. Et ce, même si elle est d'une importance incontestable dans des contextes aussi différents que la phénoménologie, la philosophie du langage, la philosophie de l'esprit ou encore dans les sciences cognitives. Que l'origine de l'emploi de cette notion, dans tous ces cas, remonte à Brentano et à son idée selon laquelle la conscience se distingue de ce qui ne l'est pas, par le fait qu'elle est toujours dirigée vers quelque chose, ne change rien à la multiplicité de ses usages. Et que l'on parle souvent comme si intentionnalité signifiait toujours la même chose ne fait que masquer ses différents usages. En vérité, il ne s'agit pas d'un concept unique. Relevant de motivations diverses, la notion d'intentionnalité joue des rôles différents dans des contextes théoriques parfois incommensurables. ${ }^{1}$

Il importe donc avant tout de préciser le sens selon lequel nous entendons cette notion et le contexte systématique dans lequel s'inscrit ce qui va suivre. Nous entendons la notion d'intentionnalité dans son sens phénoménologique et, plus précisément, dans celui qu'il a dans l'œuvre de Husserl. Certes, même dans le contexte husserlien la notion d'intentionnalité est difficile à circonscrire, et nous devrons nous résigner ici à n'en donner qu'une présentation sommaire et schématique, destinée uniquement à permettre une discussion de Spinoza.

Par contre, associer Spinoza à la notion d'intentionnalité ne relève-t-il pas d'un malentendu ? Ne s'agit-il pas d'un anachronisme, d'une problématique qui ne peut avoir de sens qu'après - et contre - toute une tradition de pensée idéaliste qui ne commence, apparemment, qu'avec Kant, c'est-à-

1. Voir l'avant propos de Janicaud, D., dir., L'intentionnalité en question. Entre phénoménologie et recherches cognitives, Paris, Vrin, 1995. 
dire bien après Spinoza $?^{2}$ Ce problème est réel même si l'on peut dire que le fait que la notion d'intentionnalité ne retrouve un droit de cité dans la pensée moderne qu'avec Brentano, n'implique pas forcément que la problématique à laquelle elle est reliée soit complètement absente de la philosophie pré-Kantienne. Husserl lui-même ne la trouve-t-il pas déjà chez Descartes ?

Il est bien connu que Husserl se réfère d'une façon essentielle à Descartes. Par contre, il ne dit presque rien de Spinoza, et ce qu'il en dit, notamment dans la Krisis, se résume à quelques généralités somme toute assez superficielles. En effet, et à quelques exceptions près (notamment Merleau-Ponty), Spinoza est quasiment absent de la littérature phénoménologique. Il semble donc que celle-ci et le spinozisme appartiennent à des mondes philosophiques trop éloignés pour susciter un rapprochement. ${ }^{3}$ Pourtant, c'est précisément un tel rapprochement que je me propose de risquer ici.

Que faut-il donc entendre par intentionnalité dans ce qui suit ? Je pense surtout à l'intentionnalité en un sens qui serait plus ou moins celui que lui donne Husserl, surtout le Husserl des Idées I, c'est-à-dire ce qui est articulé par la distinction entre noèse et noème. ${ }^{4}$ Dans cette articulation relativement tardive de sa théorie de l'intentionnalité, Husserl s'affranchit définitivement du point de départ brentanien et des discussions qui relèvent de la Gegenstandstheorie (par ex. avec Twardowski), ${ }^{5}$ ainsi que de la célèbre distinction fregéenne entre Sinn et Bedeutung. Telle qu'elle se développe vers 1913, cette théorie dépasse donc la problématique reconnue désormais comme fausse des rapports d'une représentation « interne » avec un objet « extérieur ». Elle dépasse également le terrain de la description, où l'intentionnalité est comprise comme désignant la "structure " de la conscience. Certes, la notion d'intentionnalité désigne, comme l'exprime par exemple l'article de l'Encyclopedia Britannica, le caractère fondamental de la conscience comme étant

2. L'intentionnalité est « un concept dont la fortune actuelle est immense, aussi intense que fut radicale son éclipse durant l'âge classique. Est-ce là un hasard historique ? Ou faut-il retenir l'hypothèse que prend forme dans la notion d'intentionnalité une intelligence non-idéaliste de la perception et de la connaissance, en quête de la 'chose même' ? ", Ibid. p. 7.

3. Voir Desanti, J.-T., "Spinoza et la phénoménologie ", Bloch, O., dir., Spinoza au $X X^{e}$ siècle, Paris, PUF, 1993, pp. 113-126.

4. Il s'agit donc de l'intentionnalité après le «tournant transcendantal » de Husserl. Je ne crois pourtant pas que ce "tournant " soit aussi décisif qu'on l'affirme parfois. Pour une défense de la continuité de la pensée de Husserl sur l'intentionnalité, voir Souche-Dagues, D., Le développement de l'intentionnalité dans la phénoménologie husserlienne, La Haye, Martinus Nijhoff, 1972.

5. Voir Husserl, E. \& Twardowski, K., Sur les objets intentionnels (1893-1901), Paris, Vrin, 1993 ; on consultera aussi avec profit Courtine, J.-F., " Histoire et destin phénoménologique de l'intentio ", Janicaud, D., dir., op. cit. pp. 13-36. Courtine raconte ici les origines scolastiques de l'intentionnalité brentanienne et souligne le lien entre celle-ci et l'esse objective de Duns Scot. Chez Spinoza également il y a un rapport étroit entre les deux notions. Voir Yakira, E., "Une remarque sur la question d'essence formelle et essence objective ", Bove L., dir., La recta ratio. Criticiste et spinoziste? Mélanges en l'honneur de B. Rousset, Paris, Presses de l'Université de Paris-Sorbonne, 1999, pp. 247-254. 
conscience de quelque chose. Le regard phénoménologique, poursuit Husserl, montre que cet « être dirigé » est en fait un élément essentiel et immanent des expériences conscientes, que nous pouvons par conséquent qualifier d'expériences intentionnelles. La structure directionnelle de la conscience doit donc se comprendre comme relevant d'un discours eidétique, et non pas comme un fait simplement constaté. Cette intentionnalité-là n'est donc ni une qualité formelle du discours ou de la pensée, selon laquelle ceux-ci réfèrent toujours à un objet, ni une description d'un fait (le "mental »). Ainsi qu'elle apparaît dans d'autres contextes philosophiques, la notion d'intentionnalité signifie une relation non seulement asymétrique mais aussi, pour ainsi dire, ouverte. C'est ainsi que chez Frege, par exemple, qui est à l'origine d'une bonne partie des discussions contemporaines sur cette question, le rapport entre concept et objet se comprend comme une relation d'une fonction à un argument, une relation de "saturation ». Dans ce cas l'objet n'est pas immanent à la relation d'intentionnalité dans le sens où il l'est pour l'objet intentionnel de Husserl. À titre général, la notion d'intentionnalité exprime trivialement une distinction de ce qui est conscience et de ce qui ne l'est pas, puisqu'elle donne à capter eidétiquement la conscience.

L'immanence entière de l'objet dans la pensée reçoit une articulation définitive à l'aide de la terminologie de noème et noèse. Celui-là en tant que la réalité du " contenu » pensé ; celui-ci en tant qu'un acte de penser ou, plus précisément, de viser. Penser est à la fois viser et être visé. La conscience ainsi comprise est, par son essence, ce qui engendre ou effectue le sens. Il ne s'agit évidemment pas d'un anti-réalisme quelconque, mais plutôt d'une mise en évidence de la non-pertinence de l'opposition entre "réalisme " et "antiréalisme ». Parler de la conscience comme essentiellement acte noétique et visée noématique n'est ni une description ni une explication des modalités de fonctionnement $\mathrm{du}$ " mental », mais la détermination eidétique d'une région - c'est la région du sens et de la signification, de la cohérence et de la compréhension, de l'apophantique et de la vérité. Or, ce champ déterminé par le concept intentionnalité est précisément ce que Spinoza appelait pensée.

Je soulignerai deux points pour donner substance à ce rapprochement. Le premier est que chez Spinoza la pensée et chez Husserl le champ de l'intentionnalité recouvrent, par-delà la question de la vérité, aussi celle de la raison. En effet, les chapitres III et IV de la deuxième partie des Idées 1, où la terminologie de noèse et noème est introduite, est la charnière de l'ouvrage, qui annonce la dernière partie où est abordée la question de Raison et réalité. Ainsi Husserl se pose comme un véritable successeur du rationalisme classique.

Le deuxième point est qu'on trouve chez Spinoza un parallèle intéressant et, à mon avis, non accidentel, à la noème/noèse thématique de Husserl. L'interprétation spinoziste de la relation entre une idée et son objet comme étant un rapport idealideatum relève de préoccupations qui ne sont pas entièrement étrangères à celles de Husserl. Peu avant les quelques lignes consacrées à Spinoza dans la Krisis, Husserl parle du dualisme comme étant la 
raison, comme le dit le titre de la section 11, de l'incompréhensibilité des problèmes de la raison. Il est remarquable qu'il voit en Spinoza l'auteur de la première " ontologie générale ", mais nullement un précurseur de sa propre critique du dualisme cartésien. Le monisme dont il est question chez Husserl n'est pas celui des discussions cartésiennes ou pseudo-cartésiennes sur les rapports pensée/étendue ou âme/corps. Il est plutôt le refus d'en reconnaître la pertinence. Au fond, il en est de même chez Spinoza, mais d'une façon probablement moins nette et surtout moins explicite. Le monisme spinoziste est donc beaucoup plus qu'une ontologie générale, et tout en s'inscrivant dans le contexte cartésien, il s'ouvre sur une véritable problématique de la raison.

$\mathrm{La}$ " réduction » husserlienne est une stratégie méthodologique visant la délinéation d'un champ d'immanence au sein duquel il serait possible de mener une analyse eidétique de la signification, de la cohérence et de la vérité. L'idée de l'existence d'un "monde extérieur » que la phénoménologie «met entre parenthèses » s'exprime dans les dualismes philosophiques - le dualisme extérieur/intérieur, objet/pensée, ou nature (monde)/normes transcendantes (de vérité ou de conduite). Ce sont en fait tous ces dualismes que Spinoza, autant que Husserl, réduit. On peut dire que Spinoza met en œuvre une mise entre parenthèses parallèle à celle de Husserl, sauf qu'elle s'opère dans le sens inverse. Spinoza, si l'on peut se permettre de parler ainsi, aurait donc effectué une sorte de réduction de l'Ego, de la conscience et de l'intentionnalité. Or, c'est une « mise entre parenthèses » car il ne s'agit pas d'élimination. Ainsi, de la même façon que la "réduction » husserlienne n'est pas une élimination anti-réaliste de l'objectivité de nos perceptions et de nos connaissances, mais une théorie de l'essence de l'objectivité, de même celle de Spinoza n'est pas non plus une élimination de la sphère de l'intériorité subjective, mais une théorie de son origine.

Il était question, jusqu'ici, de ce qui est commun à Spinoza et Husserl, c'est-à-dire de ce qui justifie le rapprochement entre les deux. Mais ce rapprochement ne sert qu'à mieux apprécier ce qui les sépare. En effet, ce qui me semble intéressant et philosophiquement fécond dans la démarche qui est ici la nôtre, est que sur ce fond commun, qui est un fond commun de soucis philosophiques, de rationalisme et de monisme, les théories respectives de nos deux auteurs sur la nature de la pensée sont plus ou moins opposées. Plus précisément, Spinoza proposerait une conception non-intentionnelle de la pensée, du sens et de la vérité. Il est vrai, bien évidemment, qu'il n'a pas employé la terminologie de l'intentionnalité, qui ne devient courante qu'avec Brentano, Husserl et l'école phénoménologique. Il en est de même à propos de la sophistication et du niveau de conceptualisation de la thématique en question. Pourtant, sa philosophie contient une épaisseur théorique qui devrait suffire à permettre une reconstruction philosophiquement féconde, puisqu'elle peut être lue comme une critique de l'idée selon laquelle l'intentionnalité est l'essence de la pensée et le fondement d'une théorie de la raison. 
J'ai mentionné tout à l'heure le fait que Spinoza parle des rapports idéel objet en termes des rapports idée/idéat. L'intérêt des choix terminologiques de Spinoza ne s'arrête pourtant pas là. Il y a en effet tout un champ sémantique qui comporte notamment les termes de esse formale et de esse objective, qui se rapportent d'une façon indirecte, mais tout à fait essentielle, à la problématique de l'intentionnalité. Le lien entre les deux apparait explicitement dans le Traité de la réforme de l'entendement, $\$ 33$ :

L'idée vraie (nous avons, en effet, une idée vraie) est quelque chose de différent de son idéat : car autre chose est le cercle, autre chose est l'idée du cercle. En effet, l'idée du cercle n'est pas quelque chose ayant périphérie et centre, comme le cercle, et l'idée du corps n'est pas le corps lui-même; et comme elle est quelque chose de différent de son idéat, elle sera elle-même quelque chose d'intelligible par soi ; c'est-à-dire que l'idée, quant à son essence formelle, peut être l'objet d'une autre essence objective $[\ldots . .]^{6}$

À première vue, rien de très particulier ici ; c'est l'évidence même que l'idée du cercle n'a pas de périphérie ou de centre, et que le cercle en a. Mais les choses se compliquent dès qu'on lit avec attention la suite, et qu'on s'interroge sur le sens de l'analogie que Spinoza nous offre ici : dans quel sens au juste, le rapport entre le corps et son idée peut-il être comme le rapport entre le cercle et son idée ? En effet, les choses se compliquent considérablement quand on prend en considération les propositions 11 et 13 de la deuxième partie de l'Ethique:

Prop. 11 : primum, quod actuale Mentis humanæ esse constituit, nihil aliud est, quam idea rei alicujus singularis actu existentis. ${ }^{7}$

Prop. 13 : Objectum idex humanam Mentem constituentis est Corpus, sive certus extensionis modus existens, et nihil aliud. ${ }^{8}$

L'ensemble thématique qui se dessine ici est d'une grande importance, à la fois pour la compréhension de Spinoza, et pour l'appréciation de sa place dans l'histoire de la philosophie. Sans entrer dans les détails, je me bornerai ici

6. Spinoza, B., Traité de la réforme de l'entendement, Établissement du texte, traduction, introduction et commentaires par Bernard Rousset, Paris, Vrin, 1992, p.75. Il est remarquable, et ce n'est sans doute pas un hasard, que ce paragraphe soit l'occasion de deux autres rapprochements entre Spinoza et Husserl. Desanti (art. cit.) se posant la question du quasi total silence des penseurs de l'école phénoménologique à l'égard de Spinoza, cite le habemus enim ideam veram afin de montrer l'incommensurabilité entre les deux systèmes; le commentaire de Rousset sur ce $\mathbb{S}$ (Ibid., pp.221-227) est une des rares allusions à Husserl dans la littérature sur Spinoza.

7. Spinoza. B. Ethica, (herausgegeben von Carl Gebhardt), Heidelberg, Carl Winters Universitaetsbuchhandlung, 1925, tome II, p. 94, lignes 13-15. Lorsque l'édition princeps des œuvres de Spinoza sera, dans la suite de ce texte, citée, elle le sera comme il est convenu au sein des études spinozistes : G, II, 94, 13-15, c'est-à-dire le tome II de l'édition critique de Gebhardt ci-haut mentionnée, à la page 94, lignes 13 à 15 .

8. G II, 96, 1-3. 
à dire que les deux propositions citées sont la charnière d'une mise en cause généralisée de la théorie cartésienne de la subjectivité. Il s'agit non pas, comme il apparaît parfois, d'une réduction, dans un sens de rejet, de la subjectivité, mais d'une critique principielle du rôle fondateur du sujet, ou du concept de sujet, dans une théorie de l'homme. ${ }^{9}$ Une telle critique a surtout des conséquences pour l'éthique et pour une théorie de la vie en commun des hommes, mais elle porte aussi sur des questions comme celle de la nature des rapports entre âme et corps, de la théorie de la connaissance, ou de la théorie de la raison.

L'âme n'est donc pas une substance, mais une idée. Elle ne se distingue pas, ni ontologiquement ni conceptuellement, des idées que nous disons qu'elle " possède ». L'objet de cette idée, qui est le fondement d'une âme ${ }^{10}$, est un corps, ce corps même dont nous disons qu'il est le corps qui " appartient" à cette âme. C'est plus ou moins ceci que disent les propositions que nous venons de citer. Il s'agit en effet d'une thèse d'une radicalité et d'une originalité tout à fait remarquables ; très souvent, pourtant, elle est citée ou paraphrasée plutôt qu'expliquée ou interprétée. Or, lire ces thèses spinozistes à la lumière de la problématique de l'intentionnalité devrait nous aider à mieux apprécier leur véritable valeur philosophique.

Constatons pour commencer ce qui est évident : quel que soit le rapport entre âme et corps, il est clair qu'une âme n'est pas sur son corps. Si l'âme est par essence idée, et le corps objet ou idéat - et ils le sont d'une manière paradigmatique - il est clair que le rapport idéelobjet ne peut être un rapport d'intentionnalité. Comme nous l'avons déjà vu, Husserl pense l'intentionnalité en deçà du dualisme. L'intentionnalité dont il est question ici n'est pas celle selon laquelle mots ou pensées réfèrent aux objets qui leur sont extérieurs. Cette extériorité réciproque des idées et des objets, l'opposition entre l'extériorité de l'objet et l'intériorité de la conscience, sont écartées d'emblée par Spinoza.

Or, le rejet de l'intentionnalité par Spinoza va plus loin. Car pour Spinoza, qui s'écarte ici non seulement du subjectivisme cartésien, mais aussi de toute perspective transcendantale, le rapport idée/idéat est primordial et ne suppose ni Cogito ni conscience. Comme chez Husserl, il s'agit d'une sorte de stratégie, pour ainsi dire «isolationniste ». Chez les deux penseurs, un rapport primordial entre la pensée et son contenu est censé exister indépendamment des contextes dans lesquels on l'entend d'habitude. Lorsque Husserl parle de noème et de noèse, il le fait dans le cadre de ce qu'il appelle

9. Voir Yakira, E., "Y a-t-il un sujet spinoziste ? ", Moreau, P.-F., dir., Architectures de la raison. Mélanges offerts à A. Matheron, Paris, ENS éditions Fontenay/Saint-Cloud, 1996, pp. 307-316.

10. L'existence d'une âme, dans un sens qui est pertinent pour notre discours sur l'âme humaine, présuppose apparemment une certaine complexité du corps. L'importance est que même l'âme d'un corps aussi complexe que le corps humain, ne constitue pas "un royaume dans un royaume ", autrement dit, elle n'a pas une spécificité essentielle par rapport à ce qui est 'idée' dans tous les niveaux de l'être. 
" résidu », c'est-à-dire de ce qui reste après la réduction - la conscience. Lorsque Spinoza parle d'idée et d'idéat, et d'une façon encore plus claire lorsqu'il parle d'essence objective (qui n'est que l'idée) et d'essence formelle (qui est l'objet ou, en premier chef, le corps propre), ce dont il fait abstraction est précisément la conscience. Il ne faut pas présupposer quoi que ce soit pour analyser la façon fondamentale dont une idée et son objet ne font qu'un. ${ }^{11}$ Surtout il ne faut pas présupposer une conscience, ou un agent, dont l'action, ou la structure intentionnelle serait la condition de possibilité du sens, de la signification ou de la vérité.

"Faire abstraction ", comme nous l'avons déjà dit plus d'une fois, ne signifie pas réduction (dans un sens de rejet), mais renversement de l'ordre des primautés. Le paradigme de l'argumentation de Spinoza sur cette question est donné par son petit "traité de physique ", donné après E2prop.13. Ce qu'il faut retenir de cette discussion qui, même du point de vue de la mécanique de son temps, est la naïveté même, est que l'individu corporel, qui est le véritable thème de ce "traité ", est un résultat. Le fait qu'un individu soit composé, et que la cohésion des parties qui le composent soit l'effet de causes " extérieures ", n'est pas un préjudice à son individualité. Sauf que, bien évidemment, l'individualité n'est plus un absolu. Il en est de même en ce qui concerne l'âme. D'abord parce que :

L'idée qui constitue l'être formel de l'Âme humaine n'est pas simple, mais composée d'un très grand nombre d'idées (E2prop.15).

Or, ce qui est plus intéressant est la thèse selon laquelle ce que nous appelons " conscience ", c'est-à-dire, au fond le fait de l'auto-transparence de la pensée, n'est également qu'une idée - l'idée de l'idée ou l'idée réflexive.

Les rapports entre essence objective et essence formelle, tels qu'ils sont exposés dans la deuxième partie de l'Éthique, sont ce qui donne un sens concret à la discussion plus ou moins abstraite du monisme anti-cartésien dans la première partie de l'Éthique. Le scolie de E2prop.13 le dit de la façon la plus claire :

Par ce qui précède nous ne connaissons pas seulement que l'Âme humaine est unie au corps, mais aussi ce qu'il faut entendre par l'union de l'Âme et du corps.

C'est-à-dire, que l'âme est l'idée du corps. Non pas, pourtant, dans un sens qui présuppose l'âme avant cette connaissance, mais tout au contraire, dans un sens où ce qui est $\hat{a} m e$ ne peut se comprendre qu'après l'établissement de ce qui est véritablement premier : la relation de l'idée à son idéat. Une fois articulée en termes d'essence objective et essence formelle, et une fois que cette distinction est comprise sans la position préalable d'un sujet

11. La seule présupposition, qui est la condition universelle de tout être et de l'intelligibilité de tout discours, est la substance. Cela ne change rien dans ce qui vient d'être dit. 


\section{6 · Philosophiques / Printemps 2002}

pensant, il devient clair, je pense, que cette relation n'est pas essentiellement intentionnelle.

En guise de conclusion. Pourquoi tout cela est-il intéressant ? D’abord parce que cela permet, je crois, une lecture de Spinoza qui fait apparaître la cohérence de sa pensée. Mais ce qui est peut-être encore plus intéressant, est que cela fait apparaître la profonde originalité de cette pensée, et, par là même, pose quelques questions proprement philosophiques. Je n'en mentionnerai ici qu'une. En général, la thèse de la structure intentionnelle de la conscience est comprise comme opposée à la conception représentative des rapports entre la conscience et ses objets. On connaît les grandes discussions de l'époque post-cartésienne sur cette question ; il semble parfois qu'entre les deux pôles de cette opposition se jouent toutes les possibilités d'aborder la question. Or, s'il est clair que Spinoza ne pense pas en termes de la nature représentative des idées, ${ }^{12}$ ce qui aurait réintroduit un dualisme au sein de son système, il semblerait maintenant qu'il ne pense pas non plus en termes de la nature intentionnelle des idées ou de la conscience.

12. Au moins il devrait être clair ; une opinion qui n'est pas partagée par l'auteur d'un livre récent sur Spinoza. Cf. Della Rocca, M., Representation and the Mind-Body Problem in Spinoza, New York and Oxford : Oxford University Press, 1996. 